\title{
Evaluation of NKX3.1 and C-MYC expression in canine prostatic cancer
}

\author{
Carlos Eduardo Fonseca-Alves*, Priscila Emiko Kobayashi, Renée Laufer-Amorim \\ Department of Veterinary Clinic, School of Veterinary Medicine and Animal Science, São Paulo State University - UNESP, Botucatu, SP, Brazil
}

\section{A R T I C L E I N F O}

\section{Keywords:}

Canine

Homeobox gene

Prostatic cancer

MYC

\begin{abstract}
A B S T R A C T
NKX3.1/C-MYC cross-regulation has been reported in the normal human prostate, and loss of NKX3.1 and gain of $C-M Y C$ seem to be important events in prostate cancer development and progression. The dog can be an interesting model for human prostatic disease, and yet only one previous research study has shown deregulation of NKX3.1 and MYC in the canine prostate. To address the expression of NKX3.1 and C-MYC in different canine prostatic lesions, this study verified the gene and protein expression of NKX3.1 and C-MYC in normal canine prostatic tissues. We identified a $26 \mathrm{kDa}$ band that corresponded to the NKX3.1 protein, while C-MYC showed a $50 \mathrm{kDa}$ band on Western blotting analysis of all prostatic tissues. We observed that NKX3.1 protein and transcript were down-regulated in prostate cancer (PC) samples compared with non-neoplastic samples. We also observed that C-MYC protein was overexpressed in PC samples compared with normal $(P=.001)$ and proliferative inflammatory atrophy (PIA) samples $(P=.003)$. We found a positive correlation between NKX3.1 and C-MYC protein expression in normal and PIA samples. Interestingly, a negative correlation (NKX3.1 downregulation and MYC overexpression) was observed between $N K X 3.1$ and MYC transcripts in PC. Thus, samples with higher $C$ MYC expression also exhibited higher NKX3.1 expression, which indicates the regulation of C-MYC by NKX3.1 protein. As in humans, these two genes and proteins were found to be related to canine prostate cancer. However, in contrast from what is observed in humans, in canine PC samples, the downregulation of NKX3.1 cannot be explained by DNA hypermethylation.
\end{abstract}

\section{Introduction}

Dogs are the only mammals besides humans that spontaneous develop androgen-independent prostatic carcinoma (PC) at a high frequency, and therefore, dogs are considered a natural model in which to study PC (LeRoy and Northrup, 2009; Ittmann et al., 2013). They may be used in pre-clinical studies of human PC, and research on new prognostic and predictive markers may benefit both species (LeRoy and Northrup, 2009; Alves et al., 2014). In dogs, PC is a disease with highly undifferentiated morphology and an aggressive behaviour associated with a high metastatic rate (Fonseca-Alves et al., 2015). In addition, these tumours may be independent of androgen stimulation during cancer initiation (Rivera-Calderón et al., 2016).

In human prostatic pathology, proliferative inflammatory atrophy (PIA) is a very important preneoplastic lesion that was previously described by De Marzo et al. (1999). PIA occurs as a result of chronic inflammation in the prostate gland associated with glandular atrophic proliferation (De Marzo et al., 1999). In dogs, this lesion has been described, but its role in the malignant transformation of the canine prostate is unknown (Fonseca-Alves et al., 2013; Palmieri et al., 2018). Some evidence shows that PIA has the potential to be a preneoplastic lesion in dogs, as alterations in several markers such as C-MYC and NKX3.1 (Fonseca-Alves et al., 2013), AR, PTEN, MDM2, P53 (RiveraCalderón et al., 2016), Caveolin-1, $\beta$-catenin, E-cadherin and APC (Kobayashi et al., 2018) are observed.

Genes of the homeobox family appear to have a close relationship with prostatic carcinogenesis (Thangapazham et al., 2014). In humans, NKX3.1 maps to chromosome 8p and contains a homeodomain that functions as a transcription factor. Decreased expression of $N K X 3.1$ was found to be associated with prostatic carcinogenesis (Asatiani et al., 2005). Many studies have demonstrated the importance of NKX3.1 as a tumour suppressor gene in the human prostate, and a close relationship between androgen resistance and NKX3.1 downregulation has been observed (Bowen et al., 2000; Asatiani et al., 2005; Thangapazham et al., 2014).

The $C-M Y C$ oncogene is widely studied in human cancers, and a close relationship between $C-M Y C$ copy number gains and the development of metastasis in PC has been reported (Ellwood-Yen et al., 2003; Zafarana et al., 2012). Many studies have shown MYC overexpression in prostatic intraepithelial neoplasia (PIN); however, MYC overexpression alone is not sufficient for cancer progression (Jenkins et al., 1997; Nesbit et al., 1999; Qian et al., 1997). Recently, NKX3.1/C-MYC cross-

\footnotetext{
* Corresponding author.

E-mail addresses: carloseduardofa@hotmail.com, carlos.e.alves@unesp.br (C.E. Fonseca-Alves).
} 
regulation was reported (Anderson et al., 2012). In the normal human and murine prostate, the NKX3.1 transcript regulates $C-M Y C$ and prevents its overexpression. In the carcinogenesis of human prostate cancer, NKX3.1 downregulation occurs, and consequently, C-MYC is overexpressed. NKX3.1 binds to the C-MYC gene, which demonstrates their cross- regulation (Anderson et al., 2012). In veterinary medicine, only one study reported the immunoexpression of NKX3.1 and MYC in canine prostate lesions (Fonseca-Alves et al., 2013).

Due to limited information regarding NKX3.1 and C-MYC expression in canine $\mathrm{PC}$ and the important role of these genes and proteins in human and canine PC, our research aimed to evaluate NKX3.1 and CMYC transcript and protein expression in canine prostatic tissue. In addition, DNA methylation levels in the promoter region of NKX3.1 were also evaluated to investigate the putative mechanism by which this gene is regulated.

\section{Methods}

\subsection{Ethics statement}

This study was performed in accordance with the National and International Recommendations for the Care and Use of Animals. All procedures were performed under the approval of the Animal Ethics Committee of the Faculty of Veterinary Medicine and Animal Science, UNESP, Botucatu, SP, Brazil (107/2015).

\subsection{Tissue selection}

In our study, we included twenty-four canine prostatic tissues (7 normal prostatic specimens, 7 canine PIA specimens, 7 canine PC specimens and three specimens of metastatic tissue) obtained from the Veterinary Pathology archive of the Univ. Estadual Paulista, Botucatu, SP, Brazil between 2011 and 2015. PC samples $(n=7)$ were collected during prostatectomy surgery or biopsy procedure in animals that exhibited clinical signs of PC and in those that showed signs of PC by complementary exams. PIA lesions were selected from areas close to the PC lesion. Normal samples were collected during necropsy of animals without clinical signs of prostatic disease. All prostate samples were obtained from intact dogs. For the subjects with PC, the clinical records were assessed to obtain patient information, such as treatment modalities and outcome. All samples were formalin-fixed and paraffin-embedded (FFPE), and histological classification was performed according to the method described by Lai et al. (2008). Gleason-like scores were determined according to the method of Palmieri and Grieco (2015). Frozen samples were also collected for Western blotting and pyrosequencing and were maintained at $-80^{\circ} \mathrm{C}$. Due to a lack of tissue availability, metastatic samples were evaluated only by RT-qPCR analysis.

\subsection{Western blotting}

The frozen prostate samples were sectioned in a cryostat and H\&Estained to confirm the previous diagnosis. The samples were then mechanically homogenized in $50 \mathrm{mM}$ Tris- $\mathrm{HCl}$ buffer (pH 7.5, $0.25 \%$ Triton X-100 and EDTA) using a Polytron homogenizer (Kinematica, Lucerne, Switzerland) for $30 \mathrm{~s}$ at $4^{\circ} \mathrm{C}$. Equal amounts of protein $(70 \mu \mathrm{g})$ obtained from the samples were heated at $95^{\circ} \mathrm{C}$ for $5 \mathrm{~min}$ in sampleloading buffer and were then subjected to SDS-PAGE separation or electrophoresis under reducing conditions; proteins were then transferred to nitrocellulose membranes (Sigma Chemical Co., St. Louis, MO, USA).

The blots were blocked with $6 \%$ skimmed milk in TBS-T $(10 \mathrm{mM}$ Tris-HCl pH 7.5, $150 \mathrm{mM} \mathrm{NaCl}, 0.1 \%$ Tween-20) for $2 \mathrm{~h}$ and were then probed with anti-NKX3.1 (1:300) and anti-MYC (1:800) antibodies after which the blots were incubated for $18 \mathrm{~h}$ (overnight). A goat anti- $\beta$-actin antibody (1:1000; sc-1615, Santa Cruz Biotechnology, Santa Cruz, CA,
USA) was used as a positive control. After incubation with the corresponding horseradish peroxidase-conjugated secondary antibodies, the proteins were detected by chemiluminescence (Amersham ECL Select Western Blotting Detection Reagent, GE Healthcare, Little Chalfont, United Kingdom). Protein bands were quantified by densitometry analysis and were expressed as the integrated optical density (IOD). NKX3.1 and MYC protein expression levels were normalized to the level of $\beta$-actin. Normalized data are expressed as the mean and standard deviation (SD).

\subsection{Gene expression}

The paraffin-embedded tissue samples were microdissected using 16-gauge needles. mRNA was extracted using a RecoverAll ${ }^{\mathrm{TM}}$ Total Nucleic Acid Kit (Ambion, Life Technologies, MA, USA) according to the manufacturer's instructions. The mRNA concentration was determined using a spectrophotometer (NanoDrop ${ }^{\mathrm{TM}}$, ND-8000, Thermo Scientific, MA, USA), while the mRNA integrity was evaluated using a Bioanalyzer 2100 (Agilent Technologies, CA, USA). cDNA was synthesized in a final volume of $20 \mu \mathrm{L}$, and each reaction contained $1 \mu \mathrm{g}$ of total RNA treated with DNAse I (Life Technologies, Rockville, MD, USA), 200 U of SuperScript III reverse transcriptase (Life Technologies), $4 \mu \mathrm{L}$ of $5 \times$ SuperScript First-Strand Buffer, $1 \mu \mathrm{L}$ of each dNTP at $10 \mathrm{mM}$ (Life Technologies), $1 \mu \mathrm{L}$ of Oligo-(dT)18 (500 ng/ $\mu \mathrm{L}) \quad$ (Life Technologies), $1 \mu \mathrm{L}$ of random hexamers $(100 \mathrm{ng} / \mu \mathrm{L}$ ) (Life Technologies), and $1 \mu \mathrm{L}$ of $0.1 \mathrm{M}$ DTT (Life Technologies). Reverse transcription was performed for $60 \mathrm{~min}$ at $50^{\circ} \mathrm{C}$, and the enzyme was subsequently inactivated for $15 \mathrm{~min}$ at $70^{\circ} \mathrm{C}$. cDNA was stored at $-80^{\circ} \mathrm{C}$.

RT-qPCR for NKX3.1 (Forward: 5'-TGAGGTGGTTGGAGGTTTGC-3' and Reverse: 5'-TTTCATTGGCCCATCACTGA-3'), C-MYC (Forward: 5'-GCTGCCGCTGTCACTATGG-3' and Reverse: 5'-GAACTGCTCGCGCT TCGA-3') and the endogenous genes HPRT (Forward: 5'-AGCTTGCTG GTGAAAAGGAC-3' and Reverse: 5'-TTATAGTCAAGGGCATATCC-3'), ACTB (Forward: 5'-GGCATCCTGACCCTCAAGTA-3' and Reverse: 5'-CTTCTCCATGTCGTCCCAGT-3') and PRS5 (Forward: 5'-TCACTGGT GAGAACCCCCT-3' and Reverse: 5'-CCTGATTCACACGGCGTAG- ${ }^{\prime}$ ') was performed in a total volume of $10 \mu \mathrm{L}$. The reaction mixture contained Power SYBR Green PCR Master Mix (Applied Biosystems; Foster City, CA, USA), $1 \mu \mathrm{L}$ of cDNA (1:10) and $0.3 \mu \mathrm{L}$ of each primer. The reactions were performed in triplicate in 384-well plates using QuantStudio $12 \mathrm{~K}$ Flex Thermal Cycler equipment (Applied Biosystems; Foster City, CA, USA). A dissociation curve was included in all experiments to determine the specificity of the PCR product. Relative gene expression was quantified according to the $2^{-\Delta \Delta \mathrm{CT}}$ method.

\subsection{Quantitative bisulfite pyrosequencing}

Frozen prostate samples were processed in a cryostat to confirm the previous diagnosis. DNA was extracted using a DNeasy Blood \& Tissue Kit (Qiagen, Germany) according to the manufacturer's instructions. The DNA concentration was determined using a spectrophotometer (NanoDrop $^{\mathrm{TM}}$, ND-8000, Thermo Scientific, MA, USA), while the DNA integrity was evaluated using a Bioanalyzer 2100 and an Agilent DNA 1000 Kit (Agilent Technologies, CA, USA).

We performed the bisulfite conversion of the genomic DNA using an EZ DNA Methylation-Gold Kit (Zymo Research Corporation, Irvine, CA, USA). Regions flanking the promoter region of NKX3.1 were amplified by PCR (HotStarTaq Master Mix kit - Qiagen) (Forward primer - 5' GGGATTTGTGTTTTTTGT $3^{\prime}$ and Reverse primer BIOTIN-5' ACTAATC AAAACCCCATC $3^{\prime}$ ). Pyrosequencing was performed using a sequencing primer ( $5^{\prime}$ GAATTAGTTGGAGA $3^{\prime}$ ) according to the manufacturer's instructions (PyroMark ID Q96, Qiagen and Biotage, Uppsala, Sweden). The average number of $\mathrm{CpG}$ nucleotides in each group was investigated. 


\subsection{Statistical analysis}

An ANOVA test was applied to verify the significant difference in gene expression (median RQs) among the groups (the normal, PIA, PC and metastasis groups), and a $t$-test was used to evaluate the differences between two groups. We evaluated the Spearman correlation between C-MYC and NKX3.1 transcript levels in normal, PIA and PC. A survival curve was generated only for PC using the Kaplan-Meier method, and statistical significance was determined using a log-rank test. Overall survival was defined as the period (in months) between the date of surgery and the date of death caused by the disease. We also evaluated the correlation between NKX3.1 and C-MYC transcript and protein expression. A $t$-test was used to compare protein expression (Western blotting) and methylation (pyrosequencing) between two groups. $P<.05$ was considered significant for all variables. All the statistical tests were conducted using GraphPad Prism 5 (GraphPad Software Inc., La Jolla, CA, USA).

\section{Results}

\subsection{Clinical and histopathological data}

All evaluated samples $(7 / 7)$ had a Gleason score of $10(5+5)$. The clinical information is described in Table 1. Four samples (4/7) exhibited a cribriform histological pattern (score 5), and the other three (3/7) exhibited a mixed pattern (association of cribriform - score 5 and solid patterns - score 5). Three cases presented metastasis at diagnosis. One case was a PC with a mixed pattern, and the other two cases were PC with a cribriform pattern. The most common metastatic sites were the pelvic bones and lungs $(2 / 3)$, and one case demonstrated metastasis in the iliac lymph node (1/3). All patients $(7 / 7)$ received a low dose of cyclophosphamide associated with piroxicam as a primary therapy. The median survival was 456 days (range, 342-724 days).

\subsection{Western blotting}

We identified a $26 \mathrm{kDa}$ band corresponding to the NKX3.1 protein. We observed NKX3.1 downregulation in PC samples compared with normal and PIA samples $(P=.0018)$. No significant difference was observed between normal and PIA lesions. However, a decrease in NKX3.1 expression was observed in PC compared with normal samples $(P=.0012)$ and PIA samples $(P=.001)$ (Figs. 1 and $2 \mathrm{~A})$.

MYC appeared as a $50 \mathrm{kDa}$ band in the Western blotting analysis of all prostatic tissues (Fig. 1). We observed a higher expression of MYC protein in PC samples compared with normal $(P=.001)$ and PIA samples $(P=.003)$ (Fig. 2B). We also identified a positive correlation between NKX3.1 and MYC expression in normal $(R=0.64 ; P=.0014)$ and PIA $(R=0.69 ; \mathrm{P}=.001)$ samples. Thus, samples that showed higher MYC protein expression also showed the highest NKX3.1 expression. We did not observe this correlation in the PC group. In the PC samples, all tumours exhibited high MYC protein expression and low
NKX3.1 protein expression. The Western blotting results are shown in Table 2.

\subsection{Quantitative real time $P C R$}

A gradual decrease was observed in NKX3.1 expression among normal, PIA, PC and metastasis samples. Normal samples showed the highest transcript levels, while metastatic tissues showed the lowest transcript levels (Fig. 3A). We identified a positive correlation between the gene and protein expression of NKX3.1 in normal $(R=0.7143$; $P=.001)$ and PIA samples; however, no correlation was seen in PC samples. Thus, in the PC group, NKX3.1 transcript expression was not correlated with NKX3.1 protein expression.

In contrast to what was found for NKX3.1, we identified a gradual increase in $C-M Y C$ expression among normal, PIA, PC and metastasis samples $(P=.0022)$ (Fig. 3B). Normal samples had the lowest transcript levels, and metastasis samples showed the highest transcript levels $(P=.012)$. We also found a positive correlation between C-MYC gene and protein expression. Thus, the samples with the highest transcript levels also had the highest protein levels. In PC samples, no correlation was found between C-MYC gene and protein expression. We performed a correlation analysis between the NKX3.1 and C-MYC transcript levels in normal, PIA and PC samples. Interestingly, we found a positive correlation between $N K X 3.1$ and C-MYC transcript levels in normal tissues (Spearman R: 0.6494; $P=.001$ ). However, in PC tissues, we found a negative correlation between NKX3.1 and C-MYC transcript levels (Spearman $R=-0.6630 ; P=.0014$ ). Thus, samples with the highest C-MYC expression showed the lowest NKX3.1 expression. The individual relative quantification (RQ) of $N K X 3.1$ and C-MYC genes is shown in Table 2.

\subsection{Quantitative bisulfite pyrosequencing}

We investigated NKX3.1 promoter hypermethylation as a cause of decreased gene and protein expression. We did not find a significant difference in the methylation status among normal, PIA and PC samples (Fig. 4).

\section{Discussion}

We found NKX3.1 downregulation in all PC samples; this outcome indicates that this protein is important in the development of the canine PC hypothesis that we reported earlier (Fonseca-Alves et al., 2013). Interestingly, we identified a decrease in NKX3.1 transcript levels among different types of prostatic lesions, and our results indicated that the loss of NKX3.1 is involved in prostate cancer progression and metastasis. PIA samples exhibited lower NKX3.1 transcript levels compared with normal samples, and the transcript levels were decreased further in PC and metastatic tissues. We evaluated three primary tumours and their respective metastatic lesions, and the metastatic tissues showed lower NKX3.1 transcript levels compared with the primary

Table 1

Clinical information of all canine prostatic cancers evaluated.

\begin{tabular}{|c|c|c|c|c|c|c|}
\hline Identification & Breed & Age & Metastasis $^{\mathrm{a}}$ & Histological pattern & Gleason & Treatment $^{\mathrm{b}}$ \\
\hline PC1 & MBD & 15 & Bone, Lung & Cribriform & 10 & LDMT \\
\hline PC2 & Boxer & 12 & Bone, Lung & Cribriform & 10 & LDMT \\
\hline PC3 & German Shepherd & 10 & Lung & Cribriform + Solid & 10 & LDMT \\
\hline PC4 & German Shepherd & 9 & No & Cribriform + Solid & 10 & LDMT \\
\hline PC5 & MBD & 11 & No & Cribriform & 10 & LDMT \\
\hline PC6 & Boxer & 8 & Lung & Cribriform + Solid & 10 & LDMT \\
\hline PC7 & MBD & 9 & Bone & Cribriform & 10 & LDMT \\
\hline
\end{tabular}

MBD: mixed breed dog.

${ }^{\text {a }}$ Metastasis at diagnosis.

b Low dose of metronomic chemotherapy. 


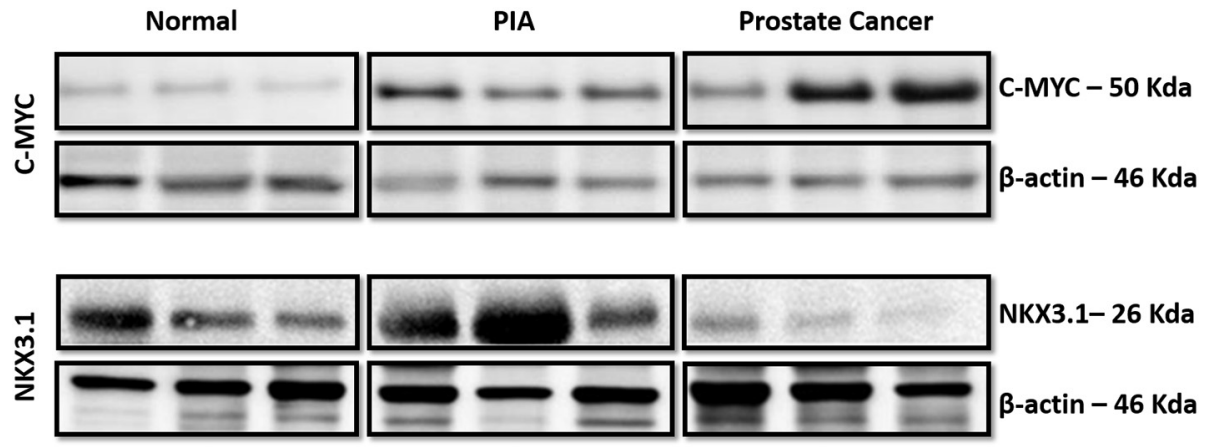

Fig. 1. Western blotting analysis of NKX3.1 and MYC proteins. Low MYC expression in normal canine tissue and an increased expression of MYC in canine prostate cancer are observed. Note the NKX3.1 downregulation compared with the normal and PIA samples.

tumour tissues. Thus, from the evaluation of the transcript levels in all groups, we identified a gradual decrease in expression (from preneoplastic lesion to metastasis). This finding implicates NKX3.1 in tumour development and metastasis. However, a limitation of this study is the small number of samples. This small number is related to the difficulty in the acquisition of fresh samples that are needed for Western blotting and pyrosequencing.

Few studies in the veterinary literature include PIA as a preneoplastic lesion, which is probably due to limitations in sample acquisition. Since PIA is an atrophic lesion, it is expected to be found only in intact dogs. Due to low levels of androgenic hormones in castrated dogs, the prostatic epithelium will atrophy, and the differentiation of atrophy due to the loss of androgenic stimulation and atrophy associated with chronic inflammation becomes impossible. In a previous study, we identified the loss of NKX3.1 in $100 \%$ of PIA samples $(22 / 22)$, which exhibited decreased immunoexpression of NKX3.1 compared with normal tissue (Fonseca-Alves et al., 2013). Herein, we confirmed NKX3.1 downregulation using two different techniques (Western blot and RT-qPCR), which indicates a role of NKX3.1 in PIA.

In human prostate cancer, the loss of NKX3.1, which occurs mainly in advanced prostate cancer (Asatiani et al., 2005), was found to be associated with a poor prognosis (Bowen et al., 2000). According to the literature, there is a consensus that canine PC is more aggressive than its human counterpart (LeRoy and Northrup, 2009). NKX3.1 downregulation could be one of the factors that contributes to the aggressive behaviour of canine PC. According to our data, metastatic tumours had lower NKX3.1 levels than non-metastatic PC.

Deletion of the $8 \mathrm{p} 21.2$ region and hypermethylation of the $N K X 3.1$ promoter have been reported in human PC as mechanisms of NKX3.1 downregulation in up to $85 \%$ of cases (Asatiani et al., 2005). Our research group investigated NKX3.1 deletion in canine PC; however, we did not find any copy number variation (CNV) changes in NKX3.1 using the array comparative genomic hybridization (aCGH) technique (data
Table 2

NKX3.1 and C-MYC protein and gene expression in all canine prostatic tissues.

\begin{tabular}{lllll}
\hline Sample ID & $\begin{array}{l}\text { NKC3.1 } \\
\text { protein }_{\text {expression }}{ }^{\mathrm{a}}\end{array}$ & $\begin{array}{l}\text { NKX3.1 gene } \\
\text { expression }^{\mathrm{b}}\end{array}$ & $\begin{array}{l}\text { C-MYC protein }_{\text {expression }^{\mathrm{a}}} \\
\text { ( }\end{array}$ & $\begin{array}{l}\text { C-MYC gene } \\
\text { expression }^{\mathrm{b}}\end{array}$ \\
\hline Nomal 1 & 2.599 & 13.09 & 0.432 & 0.01 \\
Nomal 2 & 2.325 & 15.81 & 0.453 & 0.117 \\
Nomal 3 & 1.663 & 15.58 & 0.765 & 0.442 \\
Nomal 4 & 1.096 & 15.26 & 0.687 & 1.85 \\
Nomal 5 & 0.987 & 21.933 & 0.342 & 0.346 \\
Nomal 6 & 1.304 & 19.483 & 0.283 & 0.54 \\
Nomal 7 & 1.456 & 8.526 & 0.742 & 0.07 \\
PIA 1 & 1.532 & 0.587 & 1.123 & 1.27 \\
PIA 2 & 1.85 & 3.606 & 1.198 & 0.498 \\
PIA 3 & 1.506 & 10.954 & 1.321 & 1.977 \\
PIA 4 & 2.113 & 0.422 & 1.009 & 1.25 \\
PIA 5 & 1.559 & 1.45 & 0.972 & 0.942 \\
PIA 6 & 2.249 & 2.28 & 0.877 & 3.599 \\
PIA 7 & 1.321 & 0.45 & 0.991 & 1.17 \\
PC 1 & 0.722 & 0.649 & 2.301 & 2.65 \\
PC 2 & 0.563 & 3.131 & 3.987 & 3.756 \\
PC 3 & 0.983 & 0.032 & 2.739 & 2.134 \\
PC 4 & 0.091 & 0.02 & 1.982 & 2.01 \\
PC 5 & 0.202 & 0.031 & 2.679 & 1.737 \\
PC 6 & 0.088 & 2.023 & 3.098 & 3.049 \\
PC 7 & 0.13 & 0.062 & 2.123 & 1.304 \\
Metastasis 1 & - & 0.042 & - & 2.767 \\
Metastasis 2 & - & 0.033 & - & 3.438 \\
Metastasis 3 & - & 0.03 & - & 2.880 \\
\hline
\end{tabular}

${ }^{\text {a }}$ Protein expression evaluated by Western blotting.

${ }^{\mathrm{b}}$ Relative quantification (RQ) evaluated by RT-qPCR.

not shown). Thus, we tested whether promoter hypermethylation could be a regulatory mechanism for NKX3.1 silencing in canine PC, but our methylation results did not detect hypermethylation of the NKX3.1 promoter. Other mechanisms may be important in the regulation of
A

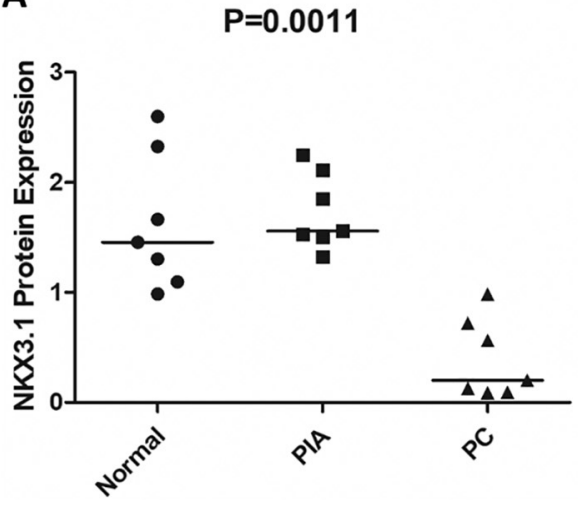

B

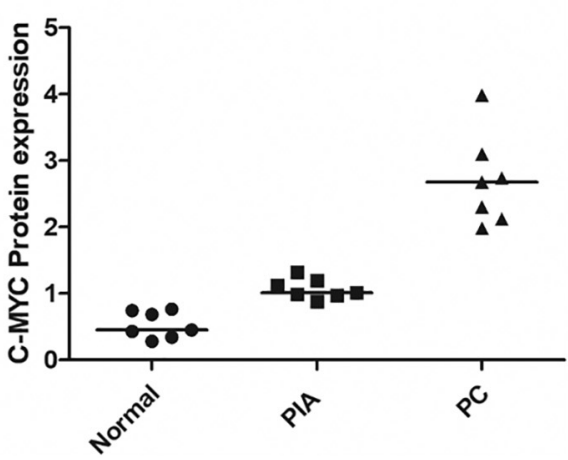

Fig. 2. Graphic representation of the Western blotting results. A: High NKX3.1 protein levels were observed in the normal and proliferative inflammatory atrophy (PIA) groups compared with the PC group. B: gradual induction of C-MYC protein expression in the normal, PIA and PC groups. PC samples exhibited higher protein expression compared with normal and PIA samples. 
A

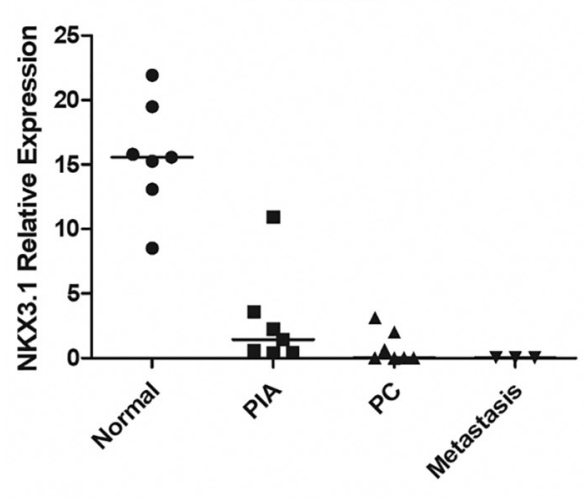

$P=0.5121$

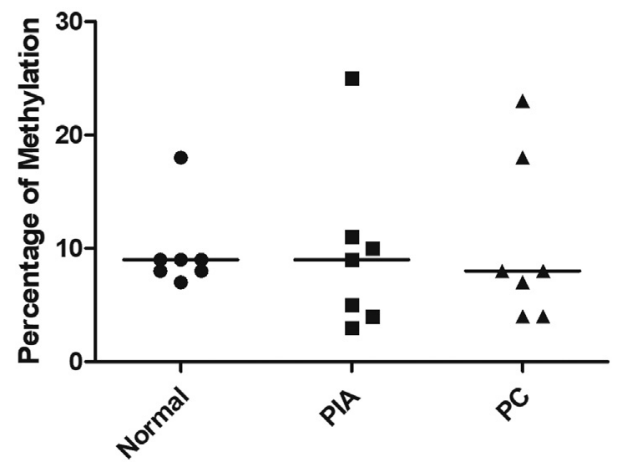

Fig. 4. Evaluation of $N K X 3.1$ promoter methylation in canine prostatic tissue. Normal, PIA and PC samples showed similar levels of methylation.

NKX3.1. According to Tan et al. (2012), NKX3.1 downregulation can be mediated by decreased expression of androgen receptor (AR). Previously, we investigated AR expression in these tumour groups and performed a correlation analysis between the protein and transcript levels of AR, but we did not find a correlation between the AR and NKX3.1 genes, which suggests post-transcriptional NKX3.1 regulation (Rivera-Calderón et al., 2016). Barnabas et al. (2011) found that the loss of NKX3.1 and a gain of the miRNA MIR151 were associated with a worse outcome in those with PC and metastatic disease. Since we did not detect NKX3.1 hypermethylation, AR regulation or CNV changes, the role of MIR151 should be investigated.

Our results showed C-MYC overexpression (gene and protein) in canine PC compared with normal and PIA samples, which indicates a gradual increase in $C-M Y C$ expression and a role in tumour progression. We identified a positive correlation between C-MYC gene and protein expression in normal prostatic tissue and in a pre-neoplastic lesion (PIA). However, no correlation was observed between $C-M Y C$ transcript levels and protein expression in canine PC. This result indicates that the C-MYC protein might be regulated by a post-transcriptional mechanism. In humans, the regulation of the C-MYC transcript by microRNA has also been reported (Yamamura et al., 2012; Misso et al., 2014).

We examined the correlation between C-MYC and NKX3.1 gene and protein expression and found a positive correlation in normal samples. Anderson et al. (2012) reported a cross-regulation between NKX3.1 and $C-M Y C$ in human PC. Those authors also examined NKX3.1 target genes and identified a fraction of the NKX3.1 gene that is a direct target of the $C-M Y C$ oncoprotein. Thus, NKX3.1 opposes C-MYC transcriptional activity. Furthermore, NKX3.1 downregulation may contribute to MYC overexpression in cancer progression (Anderson et al., 2012), as we reported in canine PC with a negative $N K X 3.1 / C-M Y C$ correlation.
$P=0.0022$

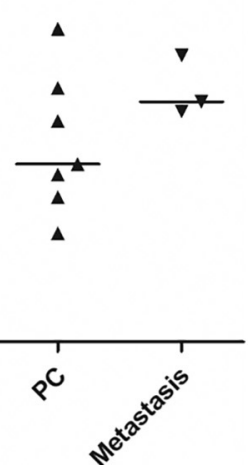

Fig. 3. A: Evaluation of $N K X 3.1$ transcript levels in the canine prostate. A gradual decrease may be observed in NKX3.1 expression. B: Evaluation of C-MYC transcript levels in canine prostatic tissue. A gradual increase in $C-M Y C$ expression may be observed among normal, PIA, PC and metastatic tissues. Metastasis samples had the highest transcript levels.

\section{Conclusion}

As in men, canine PC is influenced by the downregulation of NKX3.1 and the up regulation of C-MYC, although the mechanisms involved might be different in men since we did not observe NKX3.1 hypermethylation in our canine population. More studies should be performed in dogs to clarify the interaction between these two genes and to reveal the mechanisms involved in their abnormal expression.

\section{Authors' contributions}

The experimental design and data analyses were performed by CEFA, PEK and RLA. CEFA performed the Western blotting experiments. RLA supervised all histological and molecular procedures. CEFA wrote the manuscript draft. All authors critically reviewed the draft and approved the final manuscript.

\section{Funding}

We have received financial support from the São Paulo Research Foundation (FAPESP 2012/18426-1, FAPESP 2012/16068-0, FAPESP 2010/13774-6 and CNPq 306055/2011-2).

\section{Competing interests}

We confirm that there are no competing interests to declare.

\section{Availability of data and material} script.

All the data supporting the findings are included within the manu-

\section{Acknowledgements}

We are grateful to Dr. Marcio Carvalho for his technical support during the RT-qPCR procedures.

\section{References}

Alves, C.E.F., Busso, A.F., Silveira, S.M., Rogatto, S.R., Amorim, R.L., 2014. Abstract 5260: genomic gains in prostatic carcinoma and proliferative inflammatory atrophy in dogs. Cancer Res. 72, 5260.

Anderson, P.D., McKissic, S.A., Logan, M., Roh, M., Franco, O.E., Wang, J., Doubinskaia, I., van der Meer, R., Hayward, S.W., Eischen, C.M., Eltoum, I.E., Abdulkadir, S.A., 2012 May. Nkx3.1 and Myc cross regulate shared target genes in mouse and human prostate tumorigenesis. J. Clin. Invest. 122 (5), 1907-1919.

Asatiani, E., Huang, W.X., Wang, A., Rodriguez Ortner, E., Cavalli, L.R., Haddad, B.R., Gelmann, E.P., 2005 Feb 15. Deletion, methylation, and expression of the NKX3.1 suppressor gene in primary human prostate cancer. Cancer Res. 65 (4), 1164-1173.

Barnabas, N., Xu, L., Savera, A., Hou, Z., Barrack, E.R., 2011 Jun 1. Chromosome 8 markers of metastatic prostate cancer in African American men: gain of the MIR151 gene and loss of the NKX3-1 gene. Prostate 71 (8), 857-871. 
Bowen, C., Bubendorf, L., Voeller, H.J., Slack, R., Willi, N., Sauter, G., Gasser, T.C., Koivisto, P., Lack, E.E., Kononen, J., Kallioniemi, O.P., Gelmann, E.P., 2000 Nov 1. Loss of NKX3.1 expression in human prostate cancers correlates with tumor progression. Cancer Res. 60 (21), 6111-6115.

De Marzo, A.M., Marchi, V.L., Epstein, J.I., Nelson, W.G., 1999 Dec. Proliferative inflammatory atrophy of the prostate: implications for prostatic carcinogenesis. Am. J. Pathol. 155 (6), 1985-1992.

Ellwood-Yen, K., Graeber, T.G., Wongvipat, J., Iruela-Arispe, M.L., Zhang, J., Matusik, R., Thomas, G.V., Sawyers, C.L., 2003 Sep. Myc-driven murine prostate cancer shares molecular features with human prostate tumors. Cancer Cell 4 (3), 223-238.

Fonseca-Alves, C.E., Rodrigues, M.M., de Moura, V.M., Rogatto, S.R., Laufer-Amorim, R., 2013 Dec. Alterations of C-MYC, NKX3.1, and E-cadherin expression in canine prostate carcinogenesis. Microsc. Res. Tech. 76 (12), 1250-1256.

Fonseca-Alves, C.E., Kobayashi, P.E., Rivera-Calderón, L.G., Laufer-Amorim, R., 2015 Jun. Evidence of epithelial-mesenchymal transition in canine prostate cancer metastasis. Res. Vet. Sci. 100, 176-181.

Ittmann, M., Huang, J., Radaelli, E., Martin, P., Signoretti, S., Sullivan, R., Simons, B.W., Ward, J.M., Robinson, B.D., Chu, G.C., Loda, M., Thomas, G., Borowsky, A., Cardiff, R.D., 2013 May 1. Animal models of human prostate cancer: the consensus report of the New York meeting of the mouse models of human cancers consortium prostate pathology committee. Cancer Res. 73 (9), 2718-2736.

Jenkins, R.B., Qian, J., Lieber, M.M., Bostwick, D.G., 1997 Feb 1. Detection of c-myc oncogene amplification and chromosomal anomalies in metastatic prostatic carcinoma by fluorescence in situ hybridization. Cancer Res. 57 (3), 524-531.

Kobayashi, P.E., Fonseca-Alves, C.E., Rivera-Calderón, L.G., Carvalho, M., Kuasne, H., Rogatto, S.R., Laufer-Amorim, R., 2018, March 5. Deregulation of E-cadherin, $\beta$-catenin, APC and Caveolin-1 expression occurs in canine prostate cancer and metastatic processes. Res. Vet. Sci. 118, 254-261.

Lai, C.L., van den Ham, R., van Leenders, G., van der Lugt, J., Mol, J.A., Teske, E., 2008 Apr 1. Histopathological and immunohistochemical characterization of canine prostate cancer. Prostate 68 (5), 477-488.

Leroy, B.E., Northrup, N., 2009 May. Prostate cancer in dogs: comparative and clinical aspects. Vet. J. 180 (2), 149-162.
Misso, G., Di Martino, M.T., De Rosa, G., Farooqi, A.A., Lombardi, A., Campani, V., Zarone, M.R., Gullà, A., Tagliaferri, P., Tassone, P., Caraglia, M., 2014 Sep 23. Mir34: a new weapon against cancer? Mol. Ther. Nucleic Acids 3, e194.

Nesbit, C.E., Tersak, J.M., Prochownik, E.V., 1999 May 13. MYC oncogenes and human neoplastic disease. Oncogene 18 (19), 3004-3016.

Palmieri, C., Grieco, V., 2015 Dec. Proposal of Gleason-like grading system of canine prostate carcinoma in veterinary pathology practice. Res. Vet. Sci. 103, 11-15.

Palmieri, C., Story, M., Lean, F.Z.X., Akter, S.H., Grieco, V., de Marzo, A.M., 2018 Jan. Diagnostic utility of cytokeratin-5 for the identification of proliferative inflammatory atrophy in the canine prostate. J. Comp. Pathol. 158, 1-5.

Qian, J., Jenkins, R.B., Bostwick, D.G., 1997 Nov. Detection of chromosomal anomalies and c-myc gene amplification in the cribriform pattern of prostatic intraepithelial neoplasia and carcinoma by fluorescence in situ hybridization. Mod. Pathol. 10 (11), 1113-1119.

Rivera-Calderón, L.G., Fonseca-Alves, C.E., Kobayashi, P.E., Carvalho, M., Drigo, S.A., de Oliveira Vasconcelos, R., Laufer-Amorim, R., 2016 Jun. Alterations in PTEN, MDM2, TP53 and AR protein and gene expression are associated with canine prostate carcinogenesis. Res. Vet. Sci. 106, 56-61.

Tan, P.Y., Chang, C.W., Chng, K.R., Wansa, K.D., Sung, W.K., Cheung, E., 2012 Jan. Integration of regulatory networks by NKX3-1 promotes androgen-dependent prostate cancer survival. Mol. Cell. Biol. 32 (2), 399-414.

Thangapazham, R., Saenz, F., Katta, S., Mohamed, A.A., Tan, S.H., Petrovics, G., Srivastava, S., Dobi, A., 2014 Jan 13. Loss of the NKX3.1 tumor suppressor promotes the TMPRSS2-ERG fusion gene expression in prostate cancer. BMC Cancer 14, 16.

Yamamura, S., Saini, S., Majid, S., Hirata, H., Ueno, K., Deng, G., Dahiya, R., 2012. MicroRNA-34a modulates c-Myc transcriptional complexes to suppress malignancy in human prostate cancer cells. PLoS One 7 (1), e29722.

Zafarana, G., Ishkanian, A.S., Malloff, C.A., Locke, J.A., Sykes, J., Thoms, J., Lam, W.L., Squire, J.A., Yoshimoto, M., Ramnarine, V.R., Meng, A., Ahmed, O., Jurisica, I., Milosevic, M., Pintilie, M., van der Kwast, T., Bristow, R.G., 2012 Aug 15. Copy number alterations of c-MYC andPTEN are prognostic factors for relapse after prostate cancer radiotherapy. Cancer 118 (16), 4053-4062. 